\title{
Review Article \\ Combined Effects of Pericytes in the Tumor Microenvironment
}

\author{
Aline Lopes Ribeiro and Oswaldo Keith Okamoto \\ Centro de Pesquisa sobre o Genoma Humano e Células-Tronco, Departamento de Genética e Biologia Evolutiva, \\ Instituto de Biociências, Universidade de São Paulo, Rua do Matão 277, Cidade Universitária, 05508-090 São Paulo, SP, Brazil \\ Correspondence should be addressed to Oswaldo Keith Okamoto; keith.okamoto@usp.br
}

Received 23 January 2015; Accepted 28 February 2015

Academic Editor: Silvia Brunelli

Copyright ( 2015 A. L. Ribeiro and O. K. Okamoto. This is an open access article distributed under the Creative Commons Attribution License, which permits unrestricted use, distribution, and reproduction in any medium, provided the original work is properly cited.

\begin{abstract}
Pericytes are multipotent perivascular cells whose involvement in vasculature development is well established. Evidences in the literature also suggest that pericytes display immune properties and that these cells may serve as an in vivo reservoir of stem cells, contributing to the regeneration of diverse tissues. Pericytes are also capable of tumor homing and are important cellular components of the tumor microenvironment (TME). In this review, we highlight the contribution of pericytes to some classical hallmarks of cancer, namely, tumor angiogenesis, growth, metastasis, and evasion of immune destruction, and discuss how collectively these hallmarks could be tackled by therapies targeting pericytes, providing a rationale for cancer drugs aiming at the TME.
\end{abstract}

\section{Introduction}

It has become increasingly evident that, not only the evolving genetic aberrations in malignant cells are critical in the pathophysiology of cancer, but also the interaction among cancer cells, nonmalignant cells, soluble factors, and other elements of the tumor microenvironment (TME). In addition to cancer associated-fibroblasts, immune cells, and endothelial cells (ECs), pericytes are also one of the main cellular components of the TME, whose diverse functions in tumor initiation and progression have only been recently addressed [1].

Pericytes were first described in the 19th century, at that time named "adventitial cells" by Rouget [2]. The term "pericyte" would only be applied in 1923, by Zimmermann [3]. These cells are commonly located on microvessel walls, within the basement membrane and closely opposed to the endothelium.

Under the microscope, pericytes are typically described as highly elongated, slender, and branched cells, with projections that extend longitudinally and circumferentially around the vessel wall $[4,5]$. Pericytes have also been characterized by the expression of alpha-smooth muscle actin ( $\alpha$-SMA), desmin, CD146, platelet-derived growth factor beta receptor $(\mathrm{PDGFR} \beta)$, and nerve/glial antigen-2 (NG2) proteoglycan $[5,6]$. These markers, however, are not exclusive of pericytes and their expression may also vary according to the type of tissue, maturation stage, and pathological conditions $[5,7]$. The use of different markers or combination of markers varies in the literature and, so far, a consensus about the phenotypic identity of pericytes has not been reached. Nonetheless this issue needs to be considered to better understand pericyte biology.

For instance, in a study using double transgenic NestinGFP/NG2-DsRed mice, Birbrair et al. [8] identified two pericyte subpopulations from large blood vessels and small capillaries, named type- 1 and type- 2 pericytes. These cell subpopulations expressed common pericyte markers, such as PDGFR $\beta$, CD146, and NG2, but differed in Nestin expression. These distinct pericyte subtypes were later functionally characterized and shown to differ in their multipotent properties [8,9] and angiogenic potential [10]. In vitro and in vivo assays revealed that type-2 (Nestin-GFP+/NG2DsRed+), but not type-1 (Nestin-GFP-/NG2-DsRed+), pericytes are recruited during tumor angiogenesis. However, little is known about the ontogeny of these distinct subtypes and whether they are interconvertible.

In fact, the essential contribution of pericytes to vasculature development and maintenance has long been known. They participate in the regulation of blood flow and vessel permeability, as well as in stabilization of the vascular 
wall [11]. Pericytes also provide important mechanical and physiological support to ECs and such interaction is essential for vessel remodeling and maturation $[12,13]$. More recently, there have been growing evidences supporting new roles for pericytes in immunomodulation [14] and adult stem cell biology [15].

In the context of cancer, these distinctive pericyte properties make them important modifiers of disease progression, contributing directly or indirectly to tumor growth, metastatic spread, and resistance to therapy.

\section{Tumor Angiogenesis}

Tumor-driven angiogenesis was first described more than 100 years ago [16]. The later observation that without an efficient blood supply tumors could not grow beyond a critical size or metastasize stimulated an intensive search for pro- and antiangiogenic molecules $[17,18]$. Nowadays, some of the latest therapeutic options for treatment of different cancers rely on antiangiogenic strategies, such as bevacizumab, a monoclonal antibody targeting vascular endothelial growth factor (VEGF).

Angiogenesis is a multiple-step process tightly orchestrated by many molecules that regulate both ECs and pericytes activities. Pericytes are known to secrete growth factors that stimulate EC proliferation, in addition to proteases that contribute to modulate the surrounding extracellular matrix and guide EC migration [13, 19-21]. The proliferative endothelium from a preexisting structure with the basement membrane forms an initial tube still in an immature state. Subsequently, ECs release signals that induce pericyte recruitment [22]. The resulting pericyte coverage is crucial for vessel remodeling, maturation, and stabilization.

The reciprocal communication between ECs and pericytes is established by direct contact, by paracrine signaling, or by a newly described chemomechanical signaling pathway [23]. Some of the signaling molecules involved in this crosstalk coordination include angiopoietin-1/2 and Tie2 (Ang/Tie2), transforming growth factor- $\beta$ (TGF- $\beta$ ), and platelet-derived growth factor- $\beta$ (PDGF $\beta /$ PDGFR- $\beta$ ), which are mainly related to EC viability, mural cell differentiation, and pericyte recruitment, respectively [24].

Similar events occur during tumor angiogenesis. The sprouting of ECs is followed by a pericyte migration but, in this case, the vascular architecture does not accomplish complete maturation, which leads to several structural and functional abnormalities [25, 26]. Tumor vessels are highly disorganized, irregularly shaped, tortuous, excessively branched, and leaky [27]. The basement membrane is discontinuous or absent and presents altered composition [28]. The endothelium can be incomplete or occasionally multilayered. ECs and perivascular cells also differ functionally and morphologically from their normal counterparts [29, 30]. In tumor vessels, pericytes are loosely attached to the endothelium and exhibit cellular modifications such as differential expression of typical markers and aberrant cytoplasmic projections that invade the tumor parenchyma [31-33].

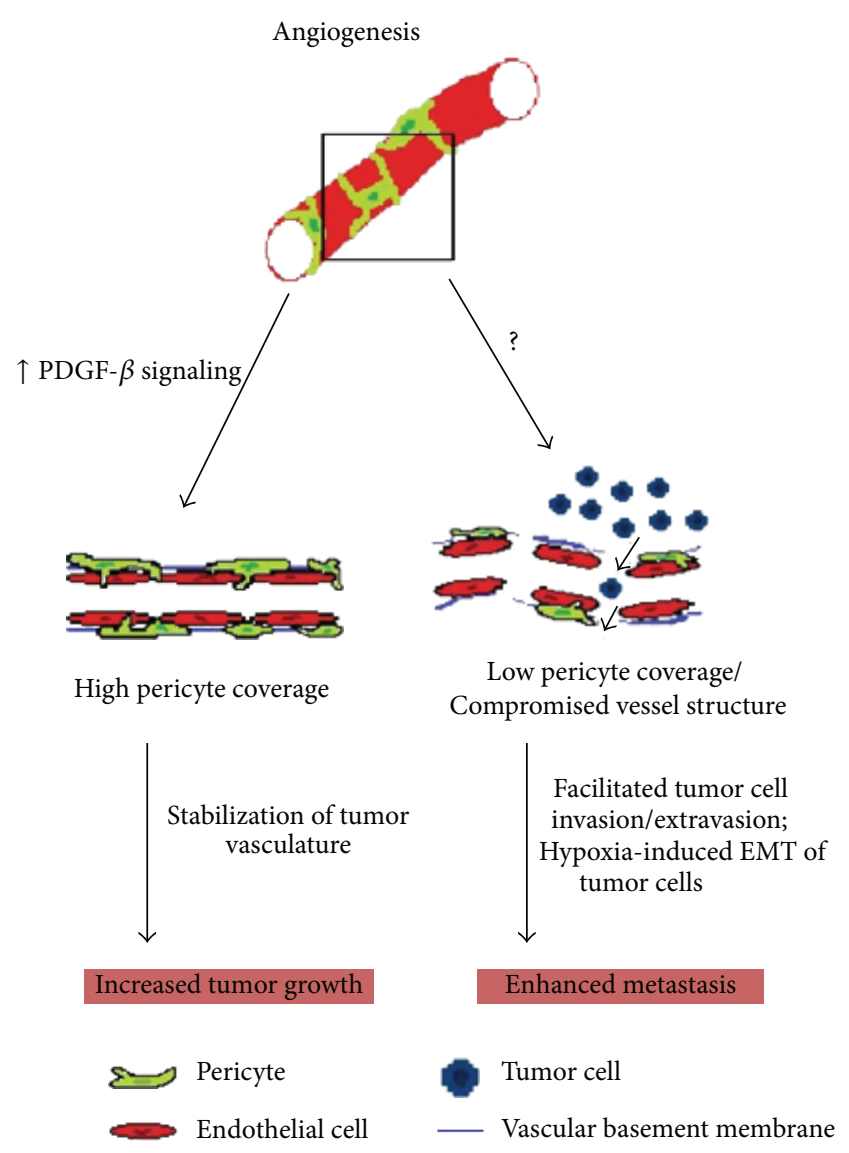

Figure 1: Abnormal pericyte coverage of tumor vessels affects tumor development. PDGF- $\beta$ signaling controls pericyte recruitment during angiogenesis. Hyperactivation of this pathway within TME may increase pericyte coverage, thereby improving vasculature stability and perfusion, which favors tumor growth. In contrast, low pericyte coverage compromises vessel structure integrity, which becomes leaky, facilitating tumor cell invasion/extravasion. Under such circumstances, tumor cells may also undergo EMT induced by hypoxia, as a consequence of lower perfusion in the tumor vasculature. Both situations enhance metastatic spread of tumor cells.

Another abnormality often observed in tumor angiogenesis is the amount of pericyte coverage on tumor vessels, ranging from high to little or no coverage at all. Clinical studies have correlated the extension of pericyte coverage on tumor microvessels with cancer prognosis [34-37]. Increased pericyte coverage has been associated with tumors of melanoma and renal cell carcinoma with aggressive clinicopathological features, resistance to therapy, and unfavorable clinical outcome of patients [38]. In contrast, pericyte dysfunction or reduction has not been correlated with prognosis. Recent studies reported that pericyte ablation leads to increased vessel permeability and poor vessel integrity which, in spite of inhibiting tumor growth, favors blood vessel invasion by tumor cells and ensuing metastatic spread $[39,40]$. These findings illustrate the many facets of pericyte effects on tumor angiogenesis (Figure 1). 
It is still unclear why tumor vessels are not able to achieve proper pericyte coverage. One important mechanism of EC-pericyte communication involves the PDGF- $\beta$ signaling, which is known to control pericyte migration during tumor angiogenesis [41]. In such mechanism, activated ECs produce PDGF- $\beta$, recruiting pericytes expressing PDGF- $\beta$ receptors $[22,42]$. In turn, pericytes stabilize the neovessels and contribute to ECs survival by locally releasing trophic factors, such as VEGF and Ang-1 [43, 44]. Blockage of pericyte recruitment by PDGF- $\beta$ pathway inhibition leads to EC loss and subsequent regression of tumor vessels [30, 45]. Overexpression of PDGF- $\beta$, on the other hand, increases pericyte coverage, improves vessel stability, and accelerates tumor growth rates $[46,47]$. Due to its relevance, therapies targeting pericyte recruitment have been considered. Other mechanisms governing pericyte migration have been covered in a recent review [48].

Pericytes have been shown to be capable of providing a scaffold of preexisting blood vessels for rapid revascularization of tumors after interruption of therapies that eliminates only ECs [49]. It seems that the remaining pericytes participate in a strategy developed by tumors to evade antiangiogenic therapies. Consequently, the combination of anti-VEGF and anti-PDGF therapies has been proposed and was shown to induce tumor vessel regression [50, 51]. More recently, treatment with anti-OLFML3 (olfactomedin-like 3) was reported to be significantly effective in reducing tumor vascularization, pericyte coverage on tumor vessels, and tumor growth [52].

Therefore, in addition to their role in tumor angiogenesis, the involvement of pericytes in vessel cooption, an important alternative pathway by which tumors obtain blood supply through the use of preexistent vessels, supports the development of novel antiangiogenic strategies targeting, not only ECs as usual, but also pericytes. The proposal that interaction of pericytes with tumor cells may determine the perivascular location of tumor propagating cells [53] provides further arguments to the relevance of pericytes in tumor development, although details of this phenomenon remain to be determined.

\section{Metastasis}

Dissemination of cancer cells to distant organs requires their survival through a challenging route beginning in the primary tumor site. Invasion into surrounding vessels or tissues, survival in a hostile environment (e.g., blood circulation), and ability to seed and recapitulate tumor growth in a new site are the main limiting steps in this process. All stages can be highly influenced by nonmalignant cells within the tumor microenvironment, including pericytes.

Although the initial studies of pericytes and tumor development were mostly focused on angiogenesis, showing that blockage of pericyte recruitment or function leads to reduced tumor growth due to compromised vessel structure and blood supply [26], later studies surprisingly revealed that loss of pericyte coverage facilitates tumor cell spreading.
One of the first evidences showing that pericytes may be negative regulators of metastasis was provide by Xian et al. (2006), using mice deficient in neural cell adhesion molecule (NCAM). In this landmark paper, they provide compelling evidence that destabilization of tumor vasculature due to detachment of pericytes and dysfunctional interaction with ECs leads to enhanced metastatic potential [54]. A previous study had already observed an enhanced metastatic frequency in knockout animals exhibiting compromised blood vessel structure [55]. Further clinical studies with colorectal and breast cancer patients corroborated this finding [35, 40]. Low pericyte coverage showed a significant correlation with distant metastasis and poorer survival. Similarly, in a xenograft model of prostate cancer, increased tumor cell invasion was associated with lower pericyte density on microvessels [56].

However, the underlying cellular and molecular mechanisms whereby pericytes may limit tumor metastasis have not been entirely elucidated. Pericytes may act as a physical barrier that makes the extravasation of tumor cells into the vessel lumen difficult and/or may actively promote metastasis by releasing factors that affect tumor invasion.

Alternatively, a recent proposal defends the idea that pericytes may be indirectly involved in tumor cell escape. They hypothesize that pericyte depletion originates leaky vessels which increases intratumoral/interstitial plasma volume and elevates local pressure. The higher fluid pressure favors compression of remaining tumor vessels, decreasing the blood flow and reinforcing hypoxia, which may trigger tumor metastasis through a hypoxia-induced epithelialmesenchymal transition (EMT) mechanism [40]. In fact, recovery of tumor vascular integrity by improving ECs junctions and increasing pericyte coverage was effective in reducing leakage and enhancing perfusion. In melanoma models, normalization of tumor vessels was able to attenuate hypoxia and decrease EMT of tumor cells, resulting in inhibition of lung and lymph node metastasis [57].

Pericytes have also been suggested to contribute to the metastatic process by affecting the colonization and growth of tumor cells at distant sites [9]. The contact of tumor cells with microvessel walls in a pericyte-like position seems to be determinant in the successful extravasation and proliferation of melanoma and lung carcinoma cells in the brain [58]. Studies with two murine models of lung metastasis showed that the administration of sunitinib, a clinically approved antiangiogenic drug, led to pericyte depletion in the seeding location [59]. Interestingly, tumor cells were preferentially retained in the lung vasculature area displaying lower pericyte coverage. The suggested hypothesis is that pericytes may limit seeding at the target site, controlling and regulating the metastatic niche.

Moreover, endothelial-derived factors have been reported to influence breast cancer cell growth at sprouting vessels in metastatic sites [60]. Taken together, these findings support the emerging idea that microvascular cells, including pericytes, may affect metastasis establishment and tumor cell growth at secondary sites. 


\section{Stemness}

The multipotent differentiation capacity of pericytes has long been proposed. In 1978, Meyrick and Reid had already demonstrated that pericytes were plastic cells, capable of developing into vascular smooth muscle cells (vSMCs) under hypoxic stress [61]. Differentiation into other nonvascular cells, primarily bone cells, was later described [62]. Subsequently, several studies described that pericytes obtained from a variety of tissues could differentiate into adipocytes, chondrocytes, and skeletal myofibers $[63,64]$. Furthermore, pericytes derived from brain capillaries were also reported to be capable of converting into neural cell lineages [65]. These and several other evidences support the hypothesis of the perivascular zone as the in vivo niche of mesenchymal stem cells (MSCs) and pericytes being the MSC precursors [66].

Indeed, besides multipotency, pericytes and MSCs share other similarities, including expression of common cellular markers. While pericytes express surface antigens typical of MSCs, such as CD44, CD73, CD90, and CD105, MSCs also express pericytes markers, including NG2, Sca-1, $\alpha$-SMA, and PDGF $\beta-\mathrm{R}$ [15], suggesting a shared ontogeny. Both cell types also present similar homing properties. Pericytes and MSCs can proliferate and migrate in response to chemotaxis and damage signals, such as those occurring during wound healing and tumor development.

Some recent findings, however, indicate that not all pericytes display stem cell potential, such as the highly differentiated pericytes found in some large and small vessels $[67,68]$. Other studies also suggest that pericytes may be a subpopulation of specialized MSC residing in perivascular locations, given that the pericytic behavior is not an intrinsic ability of all MSCs [68].

There are also growing evidences of pericytes with stemness potential in several central nervous system pathologies. Compared with other tissues, the pericyte number and coverage in brain capillaries are relatively higher, and they are crucial to the integrity and function of the blood-brain barrier. Pericytes have been considered as an alternative stem/progenitor cell reservoir within the brain, since they were shown to migrate, proliferate, and even differentiate in neural cells, in response to tissue injury, stress, and inflammation $[65,69,70]$.

In brain cancer, the perivascular niche is critical to the maintenance of a stem cell-like state in tumor cells. Interaction of perivascular cells with cancer stem cells (CSC) was shown to regulate self-renewal and differentiation of the latter cells, which are strongly related to tumor aggressiveness [71]. Notably, in Glioblastoma, the most frequent and aggressive type of primary brain tumor, a contact-dependent interaction with tumor cells, switches on the tumor-promoter character of pericytes, inducing their participation in tumor initiation and progression [53].

Another surprising connection between pericytes and brain CSC was revealed by Cheng et al. (2013) [72]. The authors demonstrated that most pericytes residing in the perivascular niche of Glioblastoma are generated by CSC. Through a close interaction with vascular components, Glioblastoma stem cells are also able to diferentiate into functional

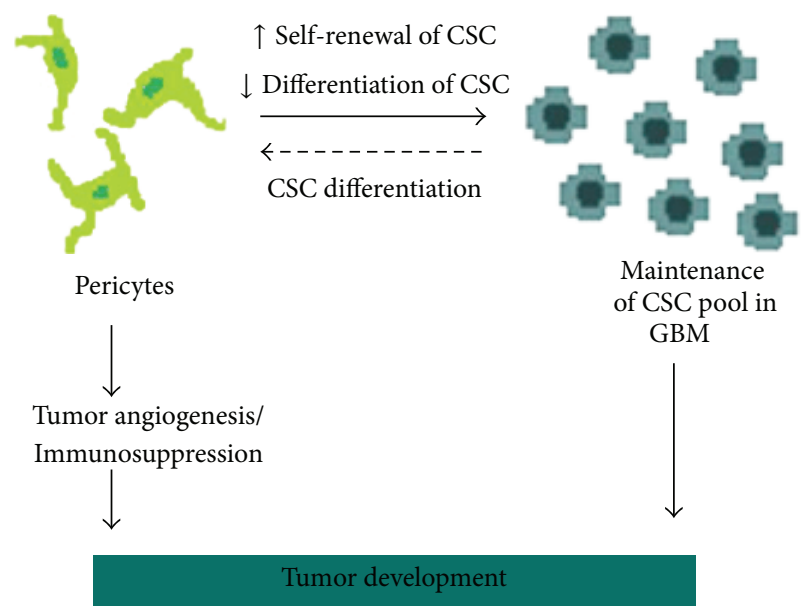

Figure 2: Interplay between pericytes and cancer stem cells. In brain cancer, the perivascular niche is critical to the maintenance of CSC pool. Perivascular cells promote self-renewal and impair differentiation of CSC. In turn, new pericytes may be generated by CSC, contributing to tumor angiogenesis and tumor scape from immune destruction. This reciprocal interaction between pericytes and CSC is highly beneficial to tumor development.

endothelial cells [73]. These findings reveal an interesting reciprocal interaction between pericytes and CSC, favoring tumor development (Figure 2).

Based on the MSC properties of pericytes, Appaix et al. [74] also proposed that neoplastic pericytes in brain capillaries could be activated and recruited in response to inflammation signals, similar to what occurs during tissue regeneration. These neoplastic pericytes would then acquire a neural stem cell-like phenotype in the brain parenchyma and generate a pool of CSC, fueling tumor development. New pericytes generated from CSC could either contribute to tumor vascularization or restart the cycle. Due to their multipotency, pericytes could also generate other stromal cells constituting the TME. In fact, pericytes have been shown to differentiate into collagen-producing fibroblasts [75] and myofibroblasts [76], two major components of the heterogeneous population of cancer-associated fibroblasts. Although plausible, further experimental evidences are needed to support this model of neoplastic pericytes as tumor initiating cells.

In addition to inflammation signals, hypoxia is another important extrinsic factor within the TME that may recruit pericytes. Interestingly, brain-derived pericytes have been recently reported to generate neurovascular cells and activated microglial cells under hypoxic conditions $[77,78]$. In gliomas, the most frequent group of primary central nervous system tumors, microglial cells are known to be recruited to the TME and activated to support tumor growth $[79,80]$. Altogether, these evidences support an important role of pericytes as precursor cells for other stromal cells within the TME.

\section{Immunomodulation}

Tumor cells can evade the immune system through different mechanisms, some of which involving multiple cellular 
components and immunosuppressive factors (e.g., TGF$\beta$, prostaglandin $\mathrm{E} 2$, and interleukin-10) from the TME. Although the contribution of pericytes in this process is still elusive, recent data support pericytes as potential targets in cancer immunotherapy approaches.

Similar to MSCs, pericytes produce cytokines, chemokines, growth factors, and adhesion molecules that regulate immune cells under certain conditions. Several genes encoding immune factors in pericytes have been reported to be upregulated by activation of the PDGF- $\beta$ signaling pathway [81], whose involvement in pericyte migration during angiogenesis was described above. In fact, pericytes have been considered an important component of the immunologic defense mechanism in the mammalian central nervous system [82, 83], where they were reported to express typical macrophage markers, such as ED-2, CD11b, CD68, and MHC class II, and exhibit immune cell properties, such as phagocytic and antigen-presentation activities [14, 84].

Brain pericytes, in particular, are highly sensitive to inflammatory stimuli and may differentially respond according to the cytokine involved. Studies with porcine brain capillary pericytes reported a rapid upregulation of iNOS and COX-2 mainly after stimulation with interleukin-1 beta (IL$1 \beta)$. Upregulation of iNOS was accompanied by increments in the intracellular oxidative status of pericytes. The same study also reported induction of phagocytosis of opsonized particles and MHC II expression in pericytes by tumor necrosis factor-alpha (TNF- $\alpha$ ) or interferon-gamma (IFN- $\gamma$ ) treatment, characteristic of an antigen-presentation activity [84].

Interaction between pericytes and immune cells also occurs during tissue repair, when pericytes may actively participate in leukocyte recruitment and diapedesis. Using an experimental model of brain inflammation, Pieper et al. [85] demonstrated that treatment with TNF- $\alpha$, IL-1 $\beta$, or LPS stimulates secretion of IL- 8 and matrix metalloprotease- 9 by brain pericytes, facilitating chemoattraction and transmigration of neutrophils.

However, in the cancer context, there are evidences that maturation of pericytes and restoration of the normal tumor vasculature improve transmigration of immune cells into tumors. A study with the RIP1-Tag5 mouse model of pancreatic islet carcinogenesis showed that deletion of the $R g s 5$ gene, encoding a regulator of G-protein signaling with expression restricted to pericytes in the vascular tissue, induced changes in the vasculature and enhanced infiltration of $\mathrm{CD}^{+} \mathrm{T}$ lymphocytes in tumors. As a consequence, the immune-mediated tumor rejection was exacerbated, resulting in improved survival of tumor-bearing mice [86].

In agreement with these observations, Bose et al. [87] reported upregulation of Rgs 5 in murine pericytes when these cells were cocultured with fragmented tumor cells or were directly injected into established tumors in vivo. Moreover, tumor-derived pericytes were able to induce CD4+ T cell anergy and this effect was rescued after Rgs5 silencing. Interestingly, in addition to Rgs5, upregulation of $P D L-1$ was also observed in pericytes cultivated in the presence of tumor fragments. Since PDL-1 expression in cancer cells is known to inhibit the activity of PD-1+/CD8+ T cells [88], the combined effects of RGS5 and PDL-1 expression in pericytes may improve protection of tumor cells from $\mathrm{T}$ cell-mediated death. Indeed, pericytes isolated from human malignant gliomas, characterized by coexpression of CD90, PDGFR- $\beta$, and CD248, were also suggested to have immunosuppressive properties within the TME, based on their capacity to inhibit proliferation of cytotoxic T lymphocytes [89].

These findings point out important direct and indirect effects of pericytes in the immune response against tumor cells, whose underlying mechanisms remain to be fully dissected.

\section{Conclusions}

An overall analysis of the functional properties of pericytes reveals that these are multifaceted cells with ability to significantly influence tumor development. As a component of the TME, pericytes may actively contribute to some classic cancer hallmarks, namely, induction of angiogenesis, sustained tumor growth, metastasis, and evasion of immune destruction.

Disruption of the delicate balance of pericyte coverage on tumor vessels seems critical since it may either induce tumor growth or facilitate metastatic spread. The interplay between pericytes and CSC is also compatible with the updated dynamic CSC model. Pericyte-mediated regulation of stemness properties in cancer cells could help maintain a residual CSC pool, whose cell progenies include both tumor and stromal cells. The immunosuppressive phenotype acquired by pericytes once in the TME is also of great relevance since they may act in synergy with tumor cells to inhibit local immune response. This scenario is highly favorable to current cancer immunotherapy strategies, such as the use of monoclonal antibodies targeting the PD-1/PDL1 signaling.

Altogether, this analysis argues in favor of pericytes as cellular targets for new cancer therapies aiming at the TME. Modern cancer treatments largely rely on such strategy, with antiangiogenic and immunosuppressive drugs as the main examples. Development of new drugs addressing pericytes would have the advantage of targeting multiple cancer hallmarks at once, increasing the chances of treatment efficacy. However, given the prometastatic effects of pericyte depletion on tumor vessels, development of such therapeutic strategy is not straightforward and should be more beneficial to earlystage diseases or to tumors with low metastatic potential. As phenotypic and functional characterization of pericytes progresses, particular subtypes of pericytes may also emerge as clearer targets for therapeutic purposes, such as the case of type-2 pericytes which are specifically recruited during tumor angiogenesis. Ultimately, pericyte-targeted therapies should be tested in combination with other treatment modalities to address possible synergistic effects aiming at meaningful tumor regression without favoring metastatic spread.

\section{Conflict of Interests}

The authors declare that they have no conflict of interests. 


\section{Acknowledgments}

This work was supported by grants from FAPESP-CEPID, CNPq, and INCT-CETGEN. Aline Lopes Ribeiro was recipient of a CAPES fellowship.

\section{References}

[1] K. Pietras and A. Östman, "Hallmarks of cancer: interactions with the tumor stroma," Experimental Cell Research, vol. 316, no. 8, pp. 1324-1331, 2010.

[2] C. Rouget, "Memoire sur le developpement, la structures et les proprieties des capillaires sanguins et lymphatiques," Archivos de Histología Normal y Patológica, vol. 5, pp. 603-633, 1873.

[3] K. W. Zimmermann, "Der feinere bau der blutcapillares," Zeitschrift für Anatomie und Entwicklungsgeschichte, vol. 68, pp. 3-109, 1923.

[4] D. E. Sims, "The pericyte-a review," Tissue \& Cell, vol. 18, no. 2, pp. 153-174, 1986.

[5] L. Díaz-Flores, R. Gutiérrez, J. F. Madrid et al., "Pericytes. Morphofunction, interactions and pathology in a quiescent and activated mesenchymal cell niche," Histology and Histopathology, vol. 24, no. 7, pp. 909-969, 2009.

[6] A. Armulik, G. Genové, and C. Betsholtz, "Pericytes: developmental, physiological, and pathological perspectives, problems, and promises," Developmental Cell, vol. 21, no. 2, pp. 193-215, 2011.

[7] R. O. Schlingemann, F. J. R. Rietveld, F. Kwaspen, P. C. M. van de Kerkhof, R. M. W. De Waal, and D. J. Ruiter, "Differential expression of markers for endothelial cells, pericytes, and basal lamina in the microvasculature of tumors and granulation tissue," American Journal of Pathology, vol. 138, no. 6, pp. 1335$1347,1991$.

[8] A. Birbrair, T. Zhang, Z.-M. Wang et al., "Role of pericytes in skeletal muscle regeneration and fat accumulation," Stem Cells and Development, vol. 22, no. 16, pp. 2298-2314, 2013.

[9] A. Birbrair, T. Zhang, Z.-M. Wang et al., "Skeletal muscle pericyte subtypes differ in their differentiation potential," Stem Cell Research, vol. 10, no. 1, pp. 67-84, 2013.

[10] A. Birbrair, T. Zhang, Z.-M. Wang, M. L. Messi, A. Mintz, and O. Delbono, "Type-1 pericytes participate in fibrous tissue deposition in aged skeletal muscle," The American Journal of Physiology-Cell Physiology, vol. 305, no. 11, pp. C1098-C1113, 2013.

[11] G. Bergers and S. Song, "The role of pericytes in blood-vessel formation and maintenance," Neuro-Oncology, vol. 7, no. 4, pp. 452-464, 2005.

[12] D. Ribatti, B. Nico, and E. Crivellato, "The role of pericytes in angiogenesis," International Journal of Developmental Biology, vol. 55, no. 3, pp. 261-268, 2011.

[13] H. Gerhardt and C. Betsholtz, "Endothelial-pericyte interactions in angiogenesis," Cell and Tissue Research, vol. 314, no. 1, pp. 15-23, 2003.

[14] R. Balabanov, R. Washington, J. Wagnerova, and P. DoreDuffy, "CNS microvascular pericytes express macrophage-like function, cell surface integrin $\alpha \mathrm{M}$, and macrophage marker ED2," Microvascular Research, vol. 52, no. 2, pp. 127-142, 1996.

[15] M. Crisan, S. Yap, L. Casteilla et al., "A perivascular origin for mesenchymal stem cells in multiple human organs," Cell Stem Cell, vol. 3, no. 3, pp. 301-313, 2008.
[16] E. Goldmann, "The growth of malignant disease in man and the lower animals with special reference to the vascular system," The Lancet, vol. 170, no. 4392, pp. 1236-1240, 1907.

[17] J. Folkman, "Tumor angiogenesis: therapeutic implications," The New England Journal of Medicine, vol. 285, no. 21, pp. 11821186, 1971.

[18] P. M. Gullino, "Angiogenesis and oncogenesis," Journal of the National Cancer Institute, vol. 61, no. 3, pp. 639-643, 1978.

[19] A. Armulik, A. Abramsson, and C. Betsholtz, "Endothelial/pericyte interactions," Circulation Research, vol. 97, no. 6, pp. 512-523, 2005.

[20] W. B. Saunders, B. L. Bohnsack, J. B. Faske et al., "Coregulation of vascular tube stabilization by endothelial cell TIMP-2 and pericyte TIMP-3," The Journal of Cell Biology, vol. 175, no. 1, pp. 179-191, 2006.

[21] P. C. Stapor, R. S. Sweat, D. C. Dashti, A. M. Betancourt, and W. L. Murfee, "Pericyte dynamics during angiogenesis: new insights from new identities," Journal of Vascular Research, vol. 51, no. 3, pp. 163-174, 2014.

[22] P. Lindblom, H. Gerhardt, S. Liebner et al., "Endothelial PDGF$B$ retention is required for proper investment of pericytes in the microvessel wall," Genes and Development, vol. 17, no. 15, pp. 1835-1840, 2003.

[23] J. T. Durham, H. K. Surks, B. M. Dulmovits, and I. M. Herman, "Pericyte contractility controls endothelial cell cycle progression and sprouting: insights into angiogenic switch mechanics," American Journal of Physiology: Cell Physiology, vol. 307, no. 9, pp. C878-C892, 2014.

[24] A. Geevarghese and I. M. Herman, "Pericyte-endothelial crosstalk: implications and opportunities for advanced cellular therapies," Translational Research, vol. 163, no. 4, pp. 296-306, 2014.

[25] U. Ozerdem and W. B. Stallcup, "Early contribution of pericytes to angiogenic sprouting and tube formation," Angiogenesis, vol. 6, no. 3, pp. 241-249, 2003.

[26] F. J. Huang, W. K. You, P. Bonaldo, T. N. Seyfried, E. B. Pasquale, and W. B. Stallcup, "Pericyte deficiencies lead to aberrant tumor vascularizaton in the brain of the NG2 null mouse," Developmental Biology, vol. 344, no. 2, pp. 1035-1046, 2010.

[27] E. Ruoslahti, "Specialization of tumour vasculature," Nature Reviews Cancer, vol. 2, no. 2, pp. 83-90, 2002.

[28] P. Baluk, S. Morikawa, A. Haskell, M. Mancuso, and D. M. McDonald, "Abnormalities of basement membrane on blood vessels and endothelial sprouts in tumors," The American Journal of Pathology, vol. 163, no. 5, pp. 1801-1815, 2003.

[29] H. Hashizume, P. Baluk, S. Morikawa et al., "Openings between defective endothelial cells explain tumor vessel leakiness," The American Journal of Pathology, vol. 156, no. 4, pp. 1363-1380, 2000.

[30] M. Hellström, H. Gerhardt, M. Kalén et al., "Lack of pericytes leads to endothelial hyperplasia and abnormal vascular morphogenesis," The Journal of Cell Biology, vol. 152, no. 3, pp. 543554, 2001.

[31] K. D. Barlow, A. M. Sanders, S. Soker, S. Ergun, and L. J. Metheny-Barlow, "Pericytes on the tumor vasculature: jekyll or hyde?" Cancer Microenvironment, vol. 6, no. 1, pp. 1-17, 2013.

[32] A. Raza, M. J. Franklin, and A. Z. Dudek, "Pericytes and vessel maturation during tumor angiogenesis and metastasis," American Journal of Hematology, vol. 85, no. 8, pp. 593-598, 2010. 
[33] S. Morikawa, P. Baluk, T. Kaidoh, A. Haskell, R. K. Jain, and D. M. McDonald, "Abnormalities in pericytes on blood vessels and endothelial sprouts in tumors," The American Journal of Pathology, vol. 160, no. 3, pp. 985-1000, 2002.

[34] Y. Cao, Z.-L. Zhang, M. Zhou et al., "Pericyte coverage of differentiated vessels inside tumor vasculature is an independent unfavorable prognostic factor for patients with clear cell renal cell carcinoma," Cancer, vol. 119, no. 2, pp. 313-334, 2013.

[35] Y. Yonenaga, A. Mori, H. Onodera et al., "Absence of smooth muscle actin-positive pericyte coverage of tumor vessels correlates with hematogenous metastasis and prognosis of colorectal cancer patients," Oncology, vol. 69, no. 2, pp. 159-166, 2005.

[36] L. Zhang, H. Nishihara, and M. R. Kano, "Pericyte-coverage of human tumor vasculature and nanoparticle permeability," Biological and Pharmaceutical Bulletin, vol. 35, no. 5, pp. 761766, 2012.

[37] M. B. O’Keeffe, A. H. Devlin, A. J. Burns et al., "Investigation of pericytes, hypoxia, and vascularity in bladder tumors: association with clinical outcomes," Oncology Research, vol. 17, no. 3, pp. 93-101, 2008.

[38] M. S. Gee, W. N. Procopio, S. Makonnen, M. D. Feldman, N. M. Yeilding, and W. M. F. Lee, "Tumor vessel development and maturation impose limits on the effectiveness of anti-vascular therapy," The American Journal of Pathology, vol. 162, no. 1, pp. 183-193, 2003.

[39] B. Sennino, B. L. Falcón, D. McCauley et al., "Sequential loss of tumor vessel pericytes and endothelial cells after inhibition of platelet-derived growth factor B by selective aptamer AX102," Cancer Research, vol. 67, no. 15, pp. 7358-7367, 2007.

[40] V. G. Cooke, V. S. LeBleu, D. Keskin et al., "Pericyte depletion results in hypoxia-associated epithelial-to-mesenchymal transition and metastasis mediated by met signaling pathway," Cancer Cell, vol. 21, no. 1, pp. 66-81, 2012.

[41] A. Abramsson, P. Lindblom, and C. Betsholtz, "Endothelial and nonendothelial sources of PDGF-B regulate pericyte recruitment and influence vascular pattern formation in tumors," Journal of Clinical Investigation, vol. 112, no. 8, pp. 1142-1151, 2003.

[42] M. Hellström, M. Kalén, P. Lindahl, A. Abramsson, and C. Betsholtz, "Role of PDGF-B and PDGFR- $\beta$ in recruitment of vascular smooth muscle cells and pericytes during embryonic blood vessel formation in the mouse," Development, vol. 126, no. 14, pp. 3047-3055, 1999 .

[43] N. Reinmuth, W. Liu, Y. D. Jung et al., "Induction of VEGF in perivascular cells defines a potential paracrine mechanism for endothelial cell survival.", The FASEB Journal, vol. 15, no. 7, pp. 1239-1241, 2001.

[44] C. Sundberg, M. Kowanetz, L. F. Brown, M. Detmar, and H. F. Dvorak, "Stable expression of angiopoietin-1 and other markers by cultured pericytes: phenotypic similarities to a subpopulation of cells in maturing vessels during later stages of angiogenesis in vivo," Laboratory Investigation, vol. 82, no. 4, pp. 387-401, 2002.

[45] P. Lindahl, B. R. Johansson, P. Levéen, and C. Betsholtz, "Pericyte loss and microaneurysm formation in PDGF-B-deficient mice," Science, vol. 277, no. 5323, pp. 242-245, 1997.

[46] M. Furuhashi, T. Sjöblom, A. Abramsson et al., "Plateletderived growth factor production by B16 melanoma cells leads to increased pericyte abundance in tumors and an associated increase in tumor growth rate," Cancer Research, vol. 64, no. 8, pp. 2725-2733, 2004.
[47] P. Guo, B. Hu, W. Gu et al., "Platelet-derived growth factor-B enhances glioma angiogenesis by stimulating vascular endothelial growth factor expression in tumor endothelia and by promoting pericyte recruitment," The American Journal of Pathology, vol. 162, no. 4, pp. 1083-1093, 2003.

[48] K. Y. Aguilera and R. A. Brekken, "Recruitment and retention: factors that affect pericyte migration," Cellular and Molecular Life Sciences, vol. 71, no. 2, pp. 299-309, 2014.

[49] M. R. Mancuso, R. Davis, S. M. Norberg et al., "Rapid vascular regrowth in tumors after reversal of VEGF inhibition," The Journal of Clinical Investigation, vol. 116, no. 10, pp. 2610-2621, 2006.

[50] G. Bergers, S. Song, N. Meyer-Morse, E. Bergsland, and D. Hanahan, "Benefits of targeting both pericytes and endothelial cells in the tumor vasculature with kinase inhibitors," Journal of Clinical Investigation, vol. 111, no. 9, pp. 1287-1295, 2003.

[51] R. Erber, A. Thurnher, A. D. Katsen et al., "Combined inhibition of VEGF and PDGF signaling enforces tumor vessel regression by interfering with pericyte-mediated endothelial cell survival mechanisms," The FASEB Journal, vol. 18, no. 2, pp. 338-340, 2004.

[52] M. Miljkovic-Licina, P. Hammel, S. Garrido-Urbani et al., “Targeting olfactomedin-like 3 inhibits tumor growth by impairing angiogenesis and pericyte coverage," Molecular Cancer Therapeutics, vol. 11, no. 12, pp. 2588-2599, 2012.

[53] E. M. Caspani, P. H. Crossley, C. Redondo-Garcia, S. Martinez, and M. G. Castro, "Glioblastoma: a pathogenic crosstalk between tumor cells and pericytes," PLOS ONE, vol. 9, no. 7, Article ID e101402, 2014.

[54] X. Xian, J. Håkansson, A. Ståhlberg et al., "Pericytes limit tumor cell metastasis," The Journal of Clinical Investigation, vol. 116, no. 3, pp. 642-651, 2006.

[55] S. Taniguchi, M. Takeoka, T. Ehara et al., "Structural fragility of blood vessels and peritoneum in calponin hl-deficient mice, resulting in an increase in hematogenous metastasis and peritoneal dissemination of malignant tumor cells," Cancer Research, vol. 61, no. 20, pp. 7627-7634, 2001.

[56] K. Welén, K. Jennbacken, T. Tešan, and J.-E. Damber, "Pericyte coverage decreases invasion of tumour cells into blood vessels in prostate cancer xenografts," Prostate Cancer and Prostatic Diseases, vol. 12, no. 1, pp. 41-46, 2009.

[57] V. Agrawal, S. Maharjan, K. Kim et al., "Direct endothelial junction restoration results in significant tumor vascular normalization and metastasis inhibition in mice," Oncotarget, vol. 5, no. 9, pp. 2761-2777, 2014.

[58] Y. Kienast, L. von Baumgarten, M. Fuhrmann et al., "Real-time imaging reveals the single steps of brain metastasis formation," Nature Medicine, vol. 16, no. 1, pp. 116-122, 2010.

[59] J. C. Welti, T. Powles, S. Foo et al., "Contrasting effects of sunitinib within in vivo models of metastasis," Angiogenesis, vol. 15, no. 4, pp. 623-641, 2012.

[60] C. M. Ghajar, H. Peinado, H. Mori et al., "The perivascular niche regulates breast tumour dormancy," Nature Cell Biology, vol. 15, no. 7, pp. 807-817, 2013.

[61] B. Meyrick and L. Reid, "The effect of continued hypoxia on rat pulmonary arterial circulation. An ultrastructural study," Laboratory Investigation, vol. 38, no. 2, pp. 188-200, 1978.

[62] K. Sato and M. R. Urist, "Induced regeneration of calvaria by bone morphogenetic protein (BMP) in dogs," Clinical Orthopaedics and Related Research, vol. 197, pp. 301-311, 1985. 
[63] C. Farrington-Rock, N. J. Crofts, M. J. Doherty, B. A. Ashton, C. Griffin-Jones, and A. E. Canfield, "Chondrogenic and adipogenic potential of microvascular pericytes," Circulation, vol. 110, no. 15, pp. 2226-2232, 2004.

[64] A. Dellavalle, M. Sampaolesi, R. Tonlorenzi et al., "Pericytes of human skeletal muscle are myogenic precursors distinct from satellite cells," Nature Cell Biology, vol. 9, no. 3, pp. 255-267, 2007.

[65] P. Dore-Duffy, A. Katychev, X. Wang, and E. van Buren, "CNS microvascular pericytes exhibit multipotential stem cell activity," Journal of Cerebral Blood Flow and Metabolism, vol. 26, no. 5, pp. 613-624, 2006.

[66] F.-J. Lv, R. S. Tuan, K. M. C. Cheung, and V. Y. L. Leung, "Concise review: the surface markers and identity of human mesenchymal stem cells," Stem Cells, vol. 32, no. 6, pp. 14081419, 2014.

[67] A. Caplan, "All MSCs are pericytes?" Cell Stem Cell, vol. 3, no. 3, pp. 229-230, 2008.

[68] A. Blocki, Y. Wang, M. Koch et al., "Not all MSCs can act as pericytes: functional in vitro assays to distinguish pericytes from other mesenchymal stem cells in angiogenesis," Stem Cells and Development, vol. 22, no. 17, pp. 2347-2355, 2013.

[69] R. Muramatsu and T. Yamashita, "Pericyte function in the physiological central nervous system," Neuroscience Research, vol. 81-82, pp. 38-41, 2014.

[70] G. Paul, I. Özen, N. S. Christophersen et al., “The adult human brain harbors multipotent perivascular mesenchymal stem cells," PLoS ONE, vol. 7, no. 4, Article ID e35577, 2012.

[71] C. Calabrese, H. Poppleton, M. Kocak et al., "A perivascular niche for brain tumor stem cells," Cancer Cell, vol. 11, no. 1, pp. 69-82, 2007.

[72] L. Cheng, Z. Huang, W. Zhou et al., "Glioblastoma stem cells generate vascular pericytes to support vessel function and tumor growth," Cell, vol. 153, no. 1, pp. 139-152, 2013.

[73] R. Wang, K. Chadalavada, J. Wilshire et al., "Glioblastoma stemlike cells give rise to tumour endothelium," Nature, vol. 468, no. 7325, pp. 829-833, 2010.

[74] F. Appaix, M. F. Nissou, B. van der Sanden et al., "Brain mesenchymal stem cells: the other stem cells of the brain?" World Journal of Stem Cells, vol. 6, no. 2, pp. 134-143, 2014.

[75] V. S. Rajkumar, K. Howell, K. Csiszar, C. P. Denton, C. M. Black, and D. J. Abraham, "Shared expression of phenotypic markers in systemic sclerosis indicates a convergence of pericytes and fibroblasts to a myofibroblast lineage in fibrosis," Arthritis Research \& Therapy, vol. 7, no. 5, pp. 1113-1123, 2005.

[76] S.-L. Lin, T. Kisseleva, D. A. Brenner, and J. S. Duffield, "Pericytes and perivascular fibroblasts are the primary source of collagen-producing cells in obstructive fibrosis of the kidney," The American Journal of Pathology, vol. 173, no. 6, pp. 1617-1627, 2008.

[77] I. Özen, T. Deierborg, K. Miharada et al., "Brain pericytes acquire a microglial phenotype after stroke," Acta Neuropathologica, vol. 128, no. 3, pp. 381-396, 2014.

[78] T. Nakagomi, S. Kubo, A. Nakano-Doi et al., "Brain vascular pericytes following ischemia have multipotential stem cell activity to differentiate into neural and vascular lineage cells," Stem Cells, 2015.

[79] G. C. Daginakatte, S. M. Gianino, N. W. Zhao, A. S. Parsadanian, and D. H. Gutmann, "Increased c-Jun-NH2-kinase signaling in neurofibromatosis-1 heterozygous microglia drives microglia activation and promotes optic glioma proliferation," Cancer Research, vol. 68, no. 24, pp. 10358-10366, 2008.
[80] A. Ellert-Miklaszewska, M. Dabrowski, M. Lipko, M. Sliwa, M. Maleszewska, and B. Kaminska, "Molecular definition of the pro-tumorigenic phenotype of glioma-activated microglia," Glia, vol. 61, no. 7, pp. 1178-1190, 2013.

[81] L. E. Olson and P. Soriano, "PDGFR $\beta$ signaling regulates mural cell plasticity and inhibits fat development," Developmental Cell, vol. 20, no. 6, pp. 815-826, 2011.

[82] E. A. Winkler, R. D. Bell, and B. V. Zlokovic, "Central nervous system pericytes in health and disease," Nature Neuroscience, vol. 14, no. 11, pp. 1398-1405, 2011.

[83] H. K. Rucker, H. J. Wynder, and W. E. Thomas, "Cellular mechanisms of CNS pericytes," Brain Research Bulletin, vol. 51, no. 5, pp. 363-369, 2000.

[84] C. Pieper, J. J. Marek, M. Unterberg, T. Schwerdtle, and H.J. Galla, "Brain capillary pericytes contribute to the immune defense in response to cytokines or LPS in vitro," Brain Research, vol. 1550, pp. 1-8, 2014.

[85] C. Pieper, P. Pieloch, and H.-J. Galla, "Pericytes support neutrophil transmigration via interleukin-8 across a porcine coculture model of the blood-brain barrier," Brain Research, vol. 1524, pp. 1-11, 2013.

[86] J. Hamzah, M. Jugold, F. Kiessling et al., "Vascular normalization in Rgs5-deficient tumours promotes immune destruction," Nature, vol. 453, no. 7193, pp. 410-414, 2008.

[87] A. Bose, S. Barik, S. Banerjee et al., "Tumor-derived vascular pericytes anergize Th cells," Journal of Immunology, vol. 191, no. 2, pp. 971-981, 2013.

[88] B. Merelli, D. Massi, L. Cattaneo, and M. Mandalà, “Targeting the PD1/PD-L1 axis in melanoma: biological rationale, clinical challenges and opportunities," Critical Reviews in Oncology/Hematology, vol. 89, no. 1, pp. 140-165, 2014.

[89] K. Ochs, F. Sahm, C. A. Opitz et al., "Immature mesenchymal stem cell-like pericytes as mediators of immunosuppression in human malignant glioma," Journal of Neuroimmunology, vol. 265, no. 1-2, pp. 106-116, 2013. 

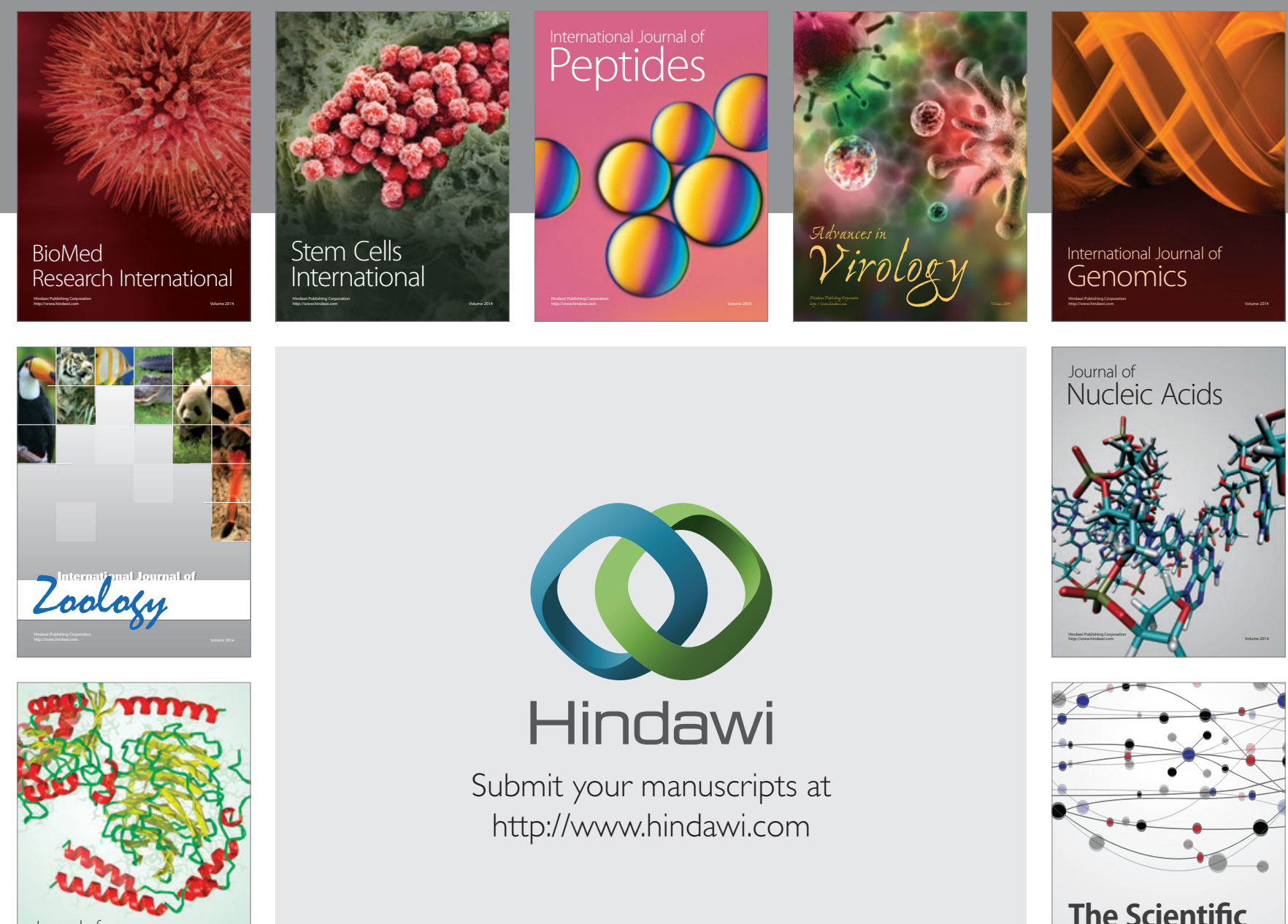

Submit your manuscripts at

http://www.hindawi.com

Journal of
Signal Transduction
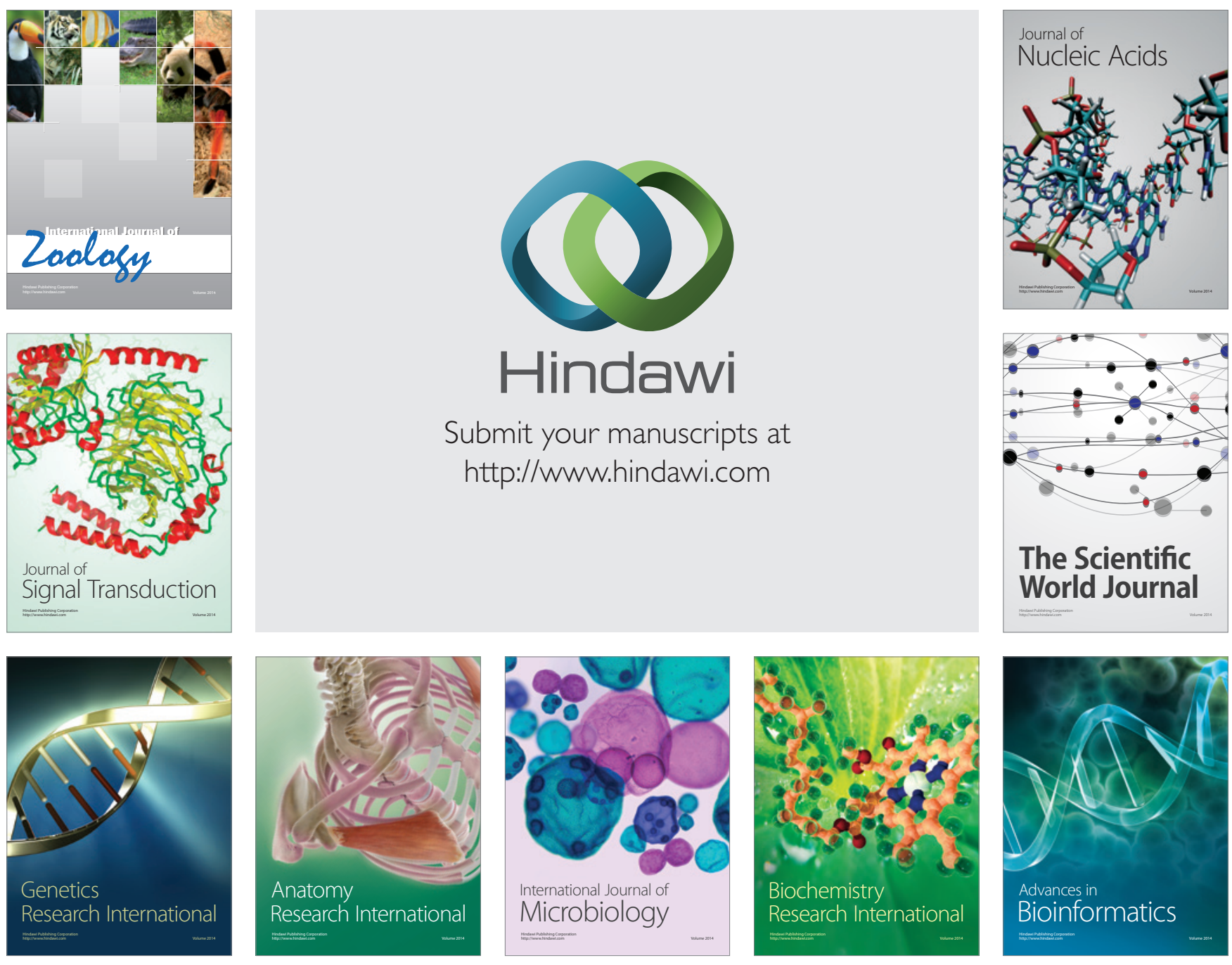

The Scientific World Journal
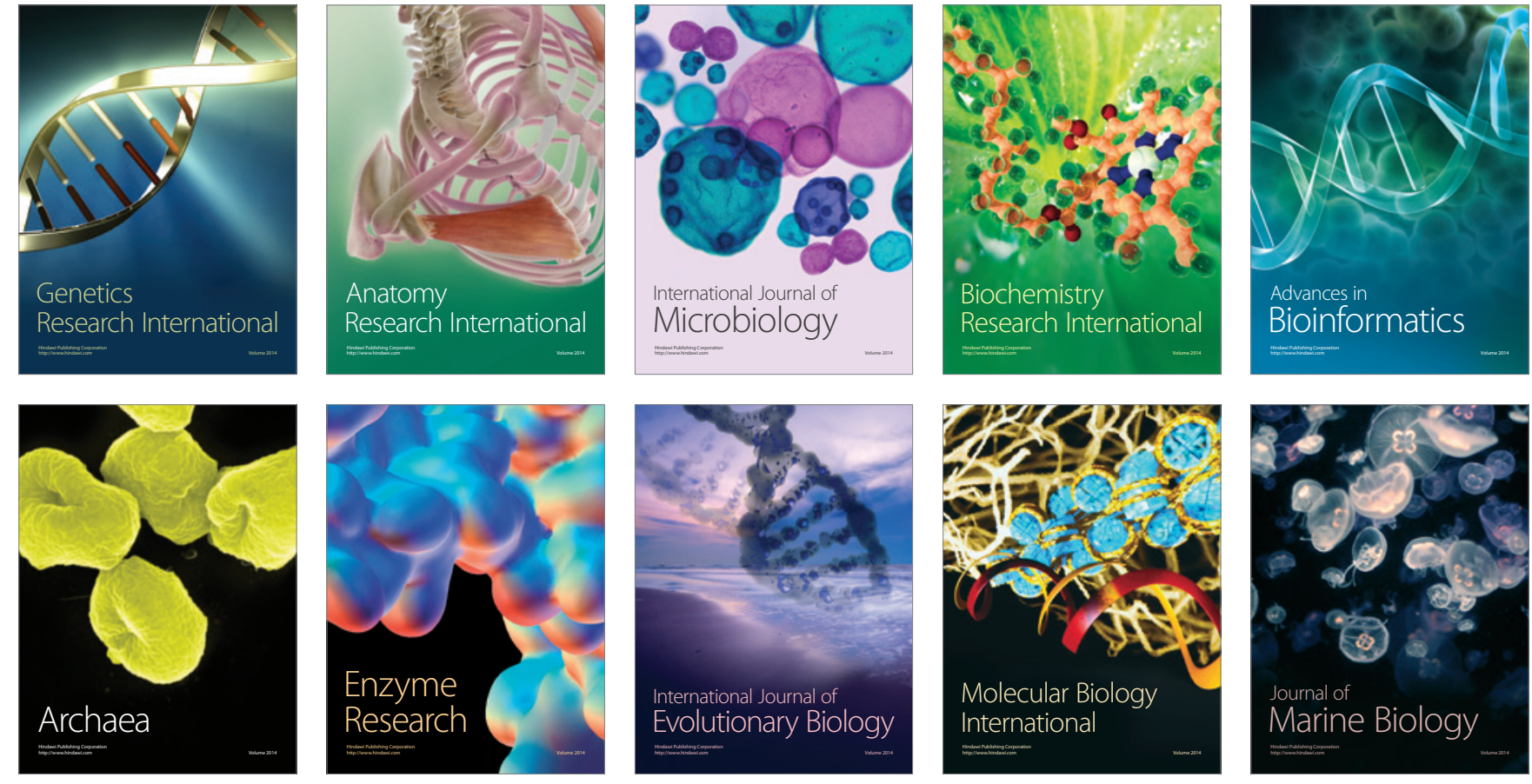